\title{
"Explosive" meridional migration of cyclones and anticyclones
}

\author{
Doron Nof ${ }^{1,2^{*}}$ \\ And \\ Stephen Van Gorder ${ }^{1}$
}

1 Dept. of Oceanography, Florida State University (FSU), Tallahassee, FL 32306

2 Also affiliated with the Geophysical Fluid Dynamics Institute (FSU)

March 14, 2007

*Corresponding Author: 419 OSB

Dept. of Oceanography

Florida State University

1015 West Call Street

Tallahassee, FL 32306

nof@ocean.fsu.edu 


\begin{abstract}
Using analytical and numerical models we examine the very rapid meridional migration induced by the barotropic component of baroclinic eddies. We show that, for the barotropic component, the main balance of forces is between the meridional $\beta$-induced force (poleward for cyclones and equatorward for anticyclones) and the opposing form-drag generated by the flow surrounding the eddy. The solution is constructed by assuming that, as the flow around a solid cylinder, the flow surrounding the eddy detaches from the eddy downstream so that there is a net form-drag exerted on the eddy. Hence, our solution is conceptually analogous to the so-called “terminal velocity" reached by objects falling in air or water where, after an initial period of acceleration, the gravitational force balances the form-drag.
\end{abstract}

We find the migration speed to be $\left(3 \pi \beta V_{m} / 5\right)^{\frac{1}{2}} r_{m}$, where $V_{m}$ is the maximum orbital speed and $r_{m}$ is the radius where the maximum speed occurs. It is typically of the order of 10 $\mathrm{km} /$ day, which is an order of magnitude faster than the classical westward speed induced by the baroclinic component $\left(\beta r_{m}{ }^{2}\right)$. The above formula gives values that are within $30-50 \%$ of the speeds measured in our own numerical experiments, and of the speeds found in numerical experiments conducted by others as well laboratory experiments.

We propose that when Agulhas rings are first formed, they most likely possess both a baroclinic component and a barotopic component underneath. Consequently, Agulhas rings first migrate to the north-northwest very fast $(10 \mathrm{~km} /$ day to the north, $1 \mathrm{~km} /$ day to the west). Cyclones with a barotropic component are not very common but, during Polymode, an extremely rapidly (northward) propagating cyclone was observed in the North Atlantic. It is proposed here that this propagation was also due to a barotropic component. 


\section{Introduction}

Numerous oceanic observations as well as numerical and analytical models show the tendency of northern hemisphere upper ocean anticyclones (cyclones) to drift to the southwest (northwest) at a rate of a few kilometers per day (e.g., Flierl, 1987; Robinson, 1983 for reviews). Traditionally, the westward component of the drift has been attributed to $\beta$ (the familiar variation of the Coriolis parameter with latitude) whereas the meridional component has been attributed to the associated radiation of energy from the light eddy to the surrounding and underlying heavier fluid. The logic behind this is that, when the orbital speed of anticyclones (cyclones) slows down due to the radiation of energy (rather than frictional decay), the now-less-energetic eddies tend to migrate toward the equator (poles) because this is where they can spin slower and yet maintain their original potential vorticity. With this conceptual picture, all eddies would ultimately reach their so-called "latitude-of-rest" where they can no longer spin at all because all of their energy has been radiated away. (In reality, eddies collide with the oceans meridional boundaries much before this happens.)

\section{a) Background}

Various numerical simulations display meridional drifts associated with the above processes (e.g., McWilliams and Flierl 1979, Mied and Liendeman 1979) and so does the analytical solution for a light lens embedded in an extremely deep slightly heavier fluid on a $\beta$ plane (Flierl, 1984). However, since in these oceanic processes the tendency of the eddies to drift westward is the main aspect of their migration and, since the meridional speed is a mere 
secondary response to this tendency, the associated meridional speeds are relatively small (a few kilometers per day), certainly not much larger than the westward speeds that created them in the first place. Although many oceanic observations do agree with these estimates, some clearly suggest that other process must also be at play on some occasions because the meridional speeds are sometimes much faster than the typical westward speeds (Fig.1). (Toward the end of their lifetime, hurricanes tend to have larger merdidional speeds than they originally possesed and, consequently, meteorologists started paying much attention to these issues in the late eighties.)

For example, very young Agulhas rings are frequently observed to migrate toward the NNW at a rate of as much as $15 \mathrm{~km} /$ day (see Fig. 1a and Byrne et al, 1995, Schmid et al. 2003), and during POLYMODE (Fig. 1b), a small cyclone was observed to propagate northward at a speed as high as $12 \mathrm{~km} /$ day (Kamenkovich et al. 1986). Both of these speeds are an order of magnitude larger than the typical westward speed (a few kilometers per day). Here, we shall suggest that, on those occasions that the meridional speeds are high, the eddies contain a strong barotropic component extending all the way from the surface to the ocean bottom. We term these very fast migrations "explosive" in order to distinguish them from the much slower "fast migrations" or "rapid waves" terms frequently used to describe the recent observations of equatorial Rossby waves in the Pacific which travel twice as fast as the theoretically predicted Rossby waves speed (see e.g., Dewar and Morris, 2000; Killworth and Blundell, 2004; Paldor et al. 2006). Also, the term "explosive" adequately describes the very temporary nature of the barotopic migrations that decay within 50-70 days. 
It is not a trivial matter to estimate the momentum introduced by the Agulahs rings to the Southeastern Atlantic and determine what fraction of the ring movement is due to an environmental flow and what is due to its very own self-propulsion. Some conventional calculations take the difference between the migration speed of each ring and the speed of its immediate environment in the Southeastern Atlantic to be the contributed anomaly. This grossly underestimates the true contributed anomaly because the volume flux of the rings (which is, say, $10 \mathrm{~Sv}$ ) is so large that the whole Southeastern Atlantic is full of remnants from old rings that also moved to the north. To correctly do the estimate, one needs to consider the motion that the Southeastern Atlantic would have had in the absence of the rings. This is the northward Sverdrup transport which is roughly $1 \mathrm{~km} /$ day, an order of magnitude smaller that the speeds of interest here. Hence, we suggest that the observed high migration speeds correspond to selfpropulsion, not the much weaker advection by the environmental flows.

We shall show that a barotropic component forces intense cyclones poleward and intense anticyclones toward the equator because these are the directions that the $\beta$-induced force is pointing to. In this scenario, the meridional $\beta$-induced force is balanced by an opposing formdrag associated with pressure exerted by the surrounding fluid flowing around the barotropic eddy. We shall neglect the effects of "lift", i.e., the sideway pressure exerted by the environmental fluid in a direction perpendicular to the direction of migration. Note that the recognition that barotropic cyclones (anticyclones) may move toward the equator (poles) was first made by Rossby (1948) who was the first to realize that all eddies are subject to a meridional $\beta$-induced force. He stopped short, however, of deriving any migratory speeds or addressing the meridional balance of driving forces. 
Dewar and Galliard (1994) also recognized the importance of a barotropic component and illustrated that, due to mutual advection (associated with the requirement that the baroclinic, and barotropic component be off-centered), a barotopic component can force rings to move rapidly in any direction. While very useful, this mechanism weakness is that it does not have a preferred direction of migration and, hence, it does not explain the observed migration which clearly has a preferred orientation. The role of bottom topography is equally questionable because the orientation of the topography varies wildly within the initial meridional path of Agulhas rings. We shall argue that the observed meridional migration is due to a balance between the $\beta$-induced force and form-drag, a very different process than the mutual-advection process of off-centered eddies or bottom topography. The force balance of our intense, nonradiating eddy is also very different from the radiation and eddy-eddy interaction issues considered by Reznik and Grimshaw (2001), Reznik et al. (2000), Reznik and Dewar (1994), Korotaev (1997), DeMaria (1985), and Firing and Beardsley (1978). As a result of the different balance, our speeds are an order of magnitude larger than that of the so-called $\beta$ gyres, as well as an order of magnitude greater than those usually discussed $\left[\sim o\left(\beta r_{m}{ }^{2}\right)\right.$, where $r_{m}$ is the radius of maximum speed ].

One of the most closely related studies to our work is that of Smith (1993), who analyzed the results of all previous numerical experiments simulating hurricanes and empirically (i.e., without addressing the associated dynamics) came up with a formula almost identical to ours. The vortices in many of the models that he analyzed were not intense and, consequently, they had a westward drift component as strong as the meridional. His formula is, nevertheless, very 
close to ours with the only difference that the coefficient $(3 \pi / 5)^{1 / 2}$ is replaced by the numerically determined coefficient of about 0.6 giving meridional speeds that are about half of ours. The difference between the two speeds is due to frictional effects that are not present in our model and yet are present in the numerics. Another very relevant study is that of Flor and Eames (2002, FE, hereafter) who carefully and meticulously measured the speeds of cyclones in the laboratory and found them to be fairly close to ours. We shall return to those two studies and their implications later. Before proceeding and describing the layout of this document, we shall briefly discuss the differences and similarities of drag and lift, both of which are critical to our study.

b) Drag-versus-lift

Both drag and lift result from asymmetrical pressure fields around the vortex-cylinder. A drag is, by definition, a pressure force in the direction opposing the migration whereas a lift is a sideway pressure force in the direction perpendicular to the migration. For many flows both forces are present and often the two are of the same order. This is certainly the case for the classical nonrotating flow around a spinning cylinder with a separation downstream. The separation causes an asymmetry in the flow direction (i.e., the downstream and upstream fields relative to the cylinder are not the same) whereas the spin introduces asymmetries in the direction perpendicular to the flow. A clockwise (counterclockwise) spin adds to the speed on the left (right) hand one side of the cylinder and subtracts from the speed on the right (left) hand side (looking downstream). In this case, the detachment causes the drag whereas the spin causes 
the lift. Without detachment, there is no drag and, without the spin, there is no lift. Note that the lift mentioned here is sometimes referred to as the "Magnus effect".

There are many flows where only one of the two forces is present. For instance, for obvious economical reasons, aeronautical engineers attempt to design (asymmetrical) wings that maximize the lift and yet minimize the drag. On the other hand, when air bubbles rise in a fluid or solid spheres fall in fluid, the flow around the object is symmetrical in the (sideway) direction perpendicular to the rise (or fall) and asymmetrical in the direction of the rise. As a result, there is only drag and no "lift" so the objects rise (or fall) exactly in the vertical direction with no movements to the sides. Often these objects reach the so-called "terminal velocity" whereby the gravitational force or buoyancy force balances the drag. (We shall see shortly that this conceptual balance is analogous to our proposed balance for the vortex.) By contrast, when an autumn leaf falls from a tree (in an otherwise still air) it moves sideways as well as downward because its shape is asymmetrical in both directions so that a horizontal pressure force (lift) is exerted on it.

For our barotropic vortex-cylinder we shall assume that there is drag but no lift. This means that the flow is taken to be symmetrical in the cross-drift direction but asymmetrical in the drift direction. The logic behind this assumption is that, in order to produce lift, the vortexcylinder must somehow transmit its very own orbital motions to the exterior fluid so that the environmental fluid will speed up on one side and slow down on the other. In the solid cylinder case, the orbital speed along the rim is large and is directly superimposed on the outer fluid in the presence of friction. On the other hand, in the vortex case, the orbital speed along its rim is 
usually zero so no motion can be easily transmitted from the vortex to the environmental fluid. It will become clear later that this no-lift assumption implies small zonal migrations, a condition that, for intense eddies, is satisfied by both our numerics and the FE experiments.

As correctly pointed out by FE, a lift produces a zonal movement, as opposed to the meridional movement that is caused by the $\beta$-force. Although FE adequately attributed the zonal movement to the lift, there estimate of the lift appears to be in disagreement with both their own experiments and with the expected velocity scales. In contrast to their calculation, their laboratory produced eddies did not accelerate in the zonal direction during the course of their runs. Also, the assumption that the maximum orbital speed is directly transmitted to the environmental fluid grossly overestimates the transmitted speed (by at least an order of magnitude) because, as just mentioned, the vortex orbital speed usually goes to zero near the outer edge. We shall return to these important points later.

\section{c) Outline}

We shall present the formulation of the problem in Section 2 beginning by showing that, conceptually, we form our anticyclones and cyclones with a barotropic component by injection or suction of upper ocean water (Figs. 2 and 3). Understandably, some readers would have preferred that we would start with the barotropic vortex right away (without going through the above process) but we believe that the injection process follows the main aspects of the actual retroflection process that is responsible for the formation of Agulhas rings in the first place. For this reason, we think that it more appropriate to describe the process in the above manner. 
As a second step, we shall approximate the above-mentioned two-layer structure by a single layer barotropic vortex with a parabolic velocity profile (Fig. 4). Since the horizontal scale of these eddies is roughly $10-100 \mathrm{~km}$, which is much smaller than the barotropic Rossby radius (roughly $2000 \mathrm{~km}$ ), we shall argue that the flow surrounding the vortices is irrotational in the sense that both the Coriolis parameter as well as the voticilty are zero. Namely, outside the vortex the flow is taken to be a potential flow, i.e., a flow whose stream function satisfies the Laplace equation. As mentioned, the familiar Magnus effect will be neglected on the ground that only small speeds are transmitted from the eddy to the surrounding fluid.

In Section 3 we show that our vortex meridional drift is in some sense analogous to the "terminal velocity" of falling or rising objects whereby buoyancy or gravity balances the from drag and the lift is zero. Here, the main vortex force balance is between the $\beta$-force induced by the vortex $\left(\beta \iint \psi d x d y\right.$ where $\psi$ is the stream function and the remaining notation is conventional) and a form-drag exerted by the fluid surrounding the vortex. This form-drag is similar to that exerted on a stationary solid cylinder by an (upstream uniform) flow that separates from the edges downstream creating low-pressure in the lee of the cylinder (relative to the highpressure on the upstream side of the cylinder). We shall see that this balance implies a meridional drift with the scale $\left(\beta V_{\max }\right)^{I / 2} r_{\max }$, where $V_{\max }$ is the vortex maximum orbital speed and $r_{\max }$ is the radius at which the maximum speed occurs.

The ratio between this meridional speed and the Rossby wave speed is $\left(V_{\max } / \beta r_{\max }^{2}\right)^{1 / 2}$, which, for typical ocean eddies, is between 10 and 100. Hence, we shall neglect the westward 
speed as well as the $\beta$-gyres and derive an analytical solution for a purely meridional drift. Since the scaling of the vortex implies that the Coriolis parameter, $f$, can be taken to be zero outside the vortex, the solution for the upstream region surrounding the vortex will be taken to be the potential flow solution for a flow surrounding a stationary cylinder (Fig. 5). As mentioned, this means that outside the eddy, the flow is a potential flow, i.e., a flow with no-vorticity and nocirculation whose stream-function satisfies the Laplace equation. Furthermore, to obtain the drift lower-bound, we shall assume that the detachment occurs at the eastern and westernmost points A and B (Fig. 5) so that the drag-force exerted on the vortex is maximized.

After obtaining the analytical solution in this manner, we shall proceed and conduct a series of numerical experiments (Section 4). The final outcome of these experiments is reasonable and a good agreement between the analytics and numerics is obtained. Our results are also in agreement with earlier numerical experiments as well as with the recent laboratory experiments of FE. However, just like in all numerical experiments of this sort, to keep the eddies (whose vorticity changes sign as one proceeds from the center to the rim) from breaking up, a non-negligible horizontal frictional coefficient has to be used. Consequently, by the time that the eddy adjusts to the presence of $\beta$ (about 60-70 days), the orbital velocity has already slowed down. Hence, the supposedly large ratio of the meridional to the zonal speed $\left(V_{\max } / \beta r_{\max }^{2}\right)^{1 / 2}$ which is $10-100$ in the ocean and of order ten in the beginning of the experiments, has been reduced to about three. This means that terms that have been neglected as small in the analytics (and are probably small in the ocean) are approximately $30 \%$ of the other terms in the numerics. This is still very reasonable and the numerical simulations display the same main 
balance that the analytics. A comparison of the analytics to the drifts observed in the ocean is presented in Section 5 where the results are summarized and discussed.

\section{Formulation}

a) Injection and suction on an f-plane

Although there is no total agreement on whether Agulhas rings are formed by the retroflection itself (Pichevin et al.1999) or some still unkown instability, it is clear that they are not formed locally by the classical meander-like instability of zonal jets. Because Agulhas rings are forced into the South Atlantic from the Indian Ocean, it is reasonable to assume that the injection process described in Fig. $\mathbf{2}$ corresponds to at least some aspects of the actual formation process. In this process, both the upper and lower rings correspond to high counterclockwise orbital speeds. Suppose, for example, that the relative vorticity of the upper ring is $0.1 f$, that the ring central depth $(\hat{h})$ is 800 meters and that it is embedded in a 4000 meter deep ocean. Under such conditions, the surface orbital speed at a radius of $100 \mathrm{~km}$ would be $0.5 \mathrm{~ms}^{-1}$ whereas the

orbital speed in the barotropic component underneath would have the scale of $f(\hat{h} / H) r$ giving about $1 \mathrm{~ms}^{-1}$. There are ways to obtain an analytical solution to this general injection problem using perturbation methods (see, e.g., Nof and Simon 1987) but such a detailed solution (which, incidentally, extends beyond the eddy boundary) is involved and is really not necessary for what we wish to do here. It is sufficient to use here a simple approximation to the more detailed injection solution. 
In line with this logic, the two eddies situated one on top of the other will be approximated by one barotropic eddy (Fig. 4) with a parabolic orbital velocity profile,

$$
V_{\theta}=-2 R_{0} f_{0} r(R-r) / R
$$

where $R_{o}$ is the Rossby number, and $R$ is the eddy radius. This speed vanishes at the center and rim and has a maximum at $R / 2$, where it has the value of $R_{0} f_{0} R / 2$. The region outside this fplane vortex is taken to be at rest.

The cyclonic eddy formation processes shown in Fig. $\mathbf{3}$ is similar to that of the anticyclone but is not exactly the same as the anticyclone formation process because the thickness along the rim of the cyclone cannot be zero. Nevertheless, in both cases it does not take much stretching of the lower layer water column to form a strong eddy underneath the light vortex. A stretching (or squeezing) of merely 500-800 meters in a $4000 \mathrm{~m}$ deep ocean is enough to produce a very strong barotropic component. Again, it is possible to obtain an approximate analytical solution to this problem but this is not necessary for the present calculation. We shall again take a parabolic profile but will now have a plus sign before (2.1).

\section{b) Scales}

With our formulation, the $\beta$-plane vortex is drifting in the north-south direction at the speed of the order of $\left(\beta V_{\max }\right)^{1 / 2} r_{\max }$ which is roughly $10 \mathrm{~cm} / \mathrm{s}$. This scale is obtained from the balance, 


$$
\beta \iint \psi d x d y \sim C^{2} r_{\max } H
$$

where $C$ is the migration rate. Relation (2.2) corresponds to a $\beta$-force (left) balanced by a formdrag (right) whose estimate is obtained by applying the Bernoulli integral to the splitting streamline associated with point 0 (Fig. 5). In a coordinate system moving with the eddycylinder, the far upstream region appears to be moving toward the eddy at $-C$ so that, by Bernoulli, the water at the stagnation point $O$ is $C^{2} / 2 g$ higher than upstream. With the aid of the potential flow solution that will be employed later, we shall see that the speed at the detachment points $\mathrm{A}$ and $\mathrm{B}$ is $2 C$ so that the wake level is $3 C^{2} / 2 g$ lower than the upstream level.

The ratio of the above speed to the familiar Rossby wave speed $\left(\beta r_{\max }{ }^{2}\right)$ is, $\left(V_{\max } / \beta r^{2}{ }_{\max }\right)^{\frac{1}{2}}$, which is typically of order ten or so. Namely, the meridional migration rate, $\left(\beta V_{\max }\right)^{1 / 2} r_{\max }$, is still much smaller than the orbital speed (which scale as $f_{0} r_{\max }$ ) because the ratio between the two speeds is $\left(\beta r_{\max } / f_{0}\right)^{1 / 2}$ which is typically 0.1 . However, at the same time, it is much faster than the Rossby wave speed. Note that, as expected, for the ocean, the ratio of the right hand side of (2.2) to the bottom stress $\left(C_{D} C V_{\max } r^{2}{ }_{\max }\right.$, where $C_{D}$ is a non-dimensional bottom drag-coefficient whose value is approximately $2 \times 10^{-3}$ ) is about ten, i.e., the form-drag is much greater than the oceanic bottom stress. Also, the form-drag is much larger than the side frictional effects induced by horizontal friction, which attempts to slow the eddy's migration down. The ratio of the form-drag (which is an inertial effect induced by friction) to the actual frictional force 
(that is acting like brakes on the eddy rim) is the Reynold's number based on the migration speed $C$, the horizontal viscosity, and the eddy radius.

Even for very slow migration rates and as high oceanic viscosity coefficient that one wishes to consider for our situation, this number is at least an order of magnitude larger than unity (in both the ocean and the numerical experiments), indicating that friction is much smaller than the terms considered in (2.2). For example, taking the migration rate to be $0.1 \mathrm{~m} / \mathrm{s}$, an eddy radius of merely $10 \mathrm{~km}$ and an oceanic horizontal eddy viscosity as high as $100 \mathrm{~m}^{2} / \mathrm{s}$, still gives a Reynolds number of 10 . However, in contrast to this oceanic situation, the Reynolds number in the numerical experiments is (unavoidably) merely three implying an error of approximately $30 \%$, and a much larger role for friction in the numerics than in the ocean.

\section{c) Methods}

To obtain the solution, we shall integrate the y-momentum equation over the vortex, i.e., over the area confined by the closed streamline separating the vortex from the surrounding fluid. It is difficult to identify such a streamline in reality (i.e., in both the numerical experiments and the ocean) because the eddy's fluid along the edge constantly mixes with the environmental fluid. In the idealized limit of no-mixing, however, such a separated streamline and an associated weak flow region in the lee of the vortex should exist as, otherwise, the $\beta$-force will forever accelerate the eddy (in the meridional direction). Such an acceleration is not observed in the ocean, laboratory, or the numerics indicating that a terminal drift velocity is reached. 
Since the meridional drift, $\left(\beta V_{\max }\right)^{1 / 2} r_{\max }$, is much slower than the orbital speed, $f_{0} r_{\max }$, we shall view the problem as a slowly varying problem. Namely, in a coordinate system slowly moving meridionally with the eddy's center (at the speed of the eddy), the problem will appear to be steady so that all the terms that explicitly involve time can be ignored. Recall that, as

mentioned, the westward drift, $\beta r_{\max }{ }^{2}$, is still much smaller than the meridional drift (which, by itself, is smaller than the orbital speed) and, consequently, it will be totally neglected. In the ocean, the three speeds have indeed three different orders of magnitude. The orbital speed is $0\left(1 \mathrm{~ms}^{-1}\right)$, the smaller meridional drift is $\sim 0\left(0.1 \mathrm{~ms}^{-1}\right)$ and the still smaller westward speed is $0\left(0.01 \mathrm{~ms}^{-1}\right)$. We shall see later that the numerics represent an approximate duplication of this velocity-ratio situation. However, it takes the numerical eddy time to adjust to $\beta$ and, by the time that this adjustment is achieved, the orbital speed $\left(V_{\max }\right)$ has been reduced and the eddy's size $\left(r_{\text {max }}\right)$ increased so that the westward speed is about a third of the meridional speed.

\section{Solution}

a) Main balance

We begin by integrating the steady $y$ - momentum equation over the drifting vortex,

$\iint\left[\partial(h u v) / \partial x+\partial\left(h v^{2}\right) / \partial y\right] d x d y+\iint f u h d x d y=g \iint(H+\eta) \partial \eta / \partial y d x d y$, 
where, in our conventional notation which is detailed in both the text and the Appendix, $\mathrm{h}$ is the total thickness, $(H+\eta), \quad u$ and $v$ the horizontal velocity components (in the meridionally moving coordinate system), $H$ the undisturbed depth, and $\eta$ is the free surface displacement $(<<H)$.

Note that the meridional migration speed $C$ does not explicitly appear in (3.1) but it is an explicit part of the x-momentum equation (which will not be used because, as we shall shortly see, it is automatically satisfied). Converting the first surface integral to a contour integral and introducing the stream function,

$$
\Psi_{y}=-u h ; \quad \Psi_{x}=v h,
$$

we obtain,

$$
\oint h u r d y-\oint h v^{2} d x+\iint\left[\beta \psi-\frac{\partial}{\partial y}(f \psi)\right] d x d y-g \oint H \eta d x=0,
$$

where we neglected terms of order of $(\eta / H)^{2}$, and the integration is done in a counterclockwise manner.

Noting further that, along any streamline such as the closed streamline separating the vortex from the environmental fluid, $u d y=v d x$, and that, without any loss of generality, we can define $\psi$ to be zero along this closed contour, (3.3) can be written as,

$$
\beta \iint \psi d x d y=g H \oint \eta d x
$$


which is our desired force balance. The left hand side is the eddy-induced $\beta$-force [southward for an anticyclone $(\Psi>0)$ and northward for the cyclone $(\Psi<0)]$ and the right hand side is the opposing form-drag resulting from a lower sea-level in the lee. Note that this balance of forces is in the direction of motion $(y)$.

Since the variations introduced by $\beta$ are small (because $\beta R / f_{o}$ is small), the left hand side of (3.4) will be computed by taking $\Psi$ to be that of the radially symmetric $f$-plane eddy described by (2.1). The right hand side of (3.4) will be computed by assuming a detachment downstream and recalling that $R$, the eddy radius, is at least an order of magnitude smaller than the barotropic Rossby radius. Therefore, the flow surrounding the eddy is not affected by rotation, i.e., it can be taken as the potential flow solution around a cylinder (i.e., a flow corresponding to no vorticity) modified by a detachment downstream.

Next, we shall use the approximated Bernoulli integral $(B)$ in the (approximately) steadily moving coordinate system,

$$
\frac{q^{2}}{2}+f_{0} C x+g \eta=B(\Psi)
$$

where, $q^{2}=u^{2}+v^{2}$, and $B$ is the Bernoulli function. The reader is referred to Nof $(1983,1985)$, where the derivation of the Bernoulli from the $x$ and $y$ momentum equations in a moving coordinate system with rotation is discussed in detail. Relation (3.5) states that the quantity, $q^{2} / 2$ $+f_{o} C x+g \eta$, is constant along a streamline (because $\psi$ is a constant along a streamline). Note that both the Magnus-lift effect (which is later neglected here) and the form-drag (which is included here) are associated with the first term on the left hand side of (3.5). 
The second term on the left hand side is associated with the so-called "planetary lift" discussed in Nof $(1983,1985)$ and will be of no consequence here as, for a barotropic fluid, it is cancelled by the Coriolis force associated with the northward movement of the eddy. To see this, we integrate the pressure term $(g \eta)$ expressed by (3.5) in $y$ over the closed vortex boundary taking into account the flow surrounding the vortex. Since the posed problem is assumed to be symmetrical in $x$ (i.e., values in $-x$ are identical to those in $+x$ ), the only term that is left from this integration (i.e., the only one that does not vanish) is the integral of the second term, $\oint f_{o} C x d y$, which represents the zonal force acting on the vortex, $\oint g \eta d y$. It is straightforward to show that, in this zonal direction, the integrated Coriolis force, acting in the opposite direction and obtained by integrating the $x$-momentum equation over the vortex interior, $\iint f_{o} C d x d y$, exactly balances this "planetary lift", $\oint f_{o} C x d y$. (Note that, as implied by the rigid lid approximation which involves the neglect of the free surface displacements everywhere except in the pressure term, we have neglected terms of order $\eta / H$ in this surface integration over the vortex interior.) This barotropic cancellation of the planetary lift (to be distinguished from the neglect of the Magnus lift) and Coriolis is not new - it was first recognized by Rossby (1948) and is mentioned in passing in his original article (again without any derivation).

\section{b) Form-drag}

To calculate the form-drag $\left(F_{D}\right)$, we will assume that, in a similar fashion to the flow surrounding a solid cylinder, the outside flow detaches from the eddy downstream. The idea behind this approach is that both a solid cylinder and an eddy correspond to a circular boundary 
through which there is no flow. The only obvious difference between the two is that, in the eddy case, the sea level is, of course, continuous across the circular boundary. Since the sea level does not enter the approximated vorticity equation (Laplace, in our case), there is no difference between the outside flow around a cylinder and that around the eddy.

The downstream detachment means that the velocities downstream are significantly smaller than those upstream so that, via Bernoulli (3.5), there is a pressure force opposing the $\beta$ force which pulls the eddy meridionally. To obtain the lower bound on the migration speed, we will assume that detachments occur at the easternmost and westernmost points (A and B in Fig. 5) so that the form-drag is maximized. Upstream (and only upstream) of AOB, the flow is taken to be the potential flow solution around a solid cylinder. [Acheson (1990) describes a similar approach for a slightly different form-drag calculation.] According to that solution, the stream function (in polar coordinates, $r$ and $\theta$, where $\theta$ is measured counterclockwise from the $x$ axis) satisfying the boundary conditions,

$$
\Psi \rightarrow-C r \cos \theta, \text { at } r \rightarrow \infty \text { and, } \Psi=0 \text { at } r=R
$$

is,

$$
\Psi=C\left(r-R^{2} / r\right) \cos \theta
$$

It corresponds to,

$$
u_{r}=C\left(1-R^{2} / r^{2}\right) \cos \theta, \text { and } u_{\theta}=-C\left(1+R^{2} / r^{2}\right) \cos \theta
$$


where $u_{r}$ and $u_{\theta}$ are the radial and tangential velocities. Accordingly, the speed $(q)$ along the vortex edge (i.e., the streamline separating the cylinder-vortex from the surrounding fluid, where $r=R)$ is,

$$
q=-2 C \cos \theta
$$

where, as mentioned, $\theta=0$ along the positive $\mathrm{x}$ axis. Recall that, when there is no detachment downstream, this potential flow is symmetrical with respect to both the $x$ and $y$ axes implying that there is no net force on the cylinder--eddy. This is the so-called d'Alembert Paradox.

Taking the flow downstream of the detachment points to be zero and considering Bernoulli (3.5), the form-drag $\left(F_{D}\right)$ is found by integrating the pressure $(g \eta)$ around the eddy (in $x)$ to be,

$$
F_{D}=g H \oint \eta d x=H R \int_{0}^{\pi}\left(q^{2} / 2\right) \sin \theta d \theta=(4 / 3) H R C^{2}
$$

Note that all the other terms in (3.5) vanish upon the integration and that $F_{D}$ is always opposing the migration.

c) The $\beta$-force

The force pulling the vortex merdionally, $F_{\beta}$, is,

$$
F_{\beta}=\int_{0}^{R} \int_{0}^{2 \pi} \psi r d \theta d r
$$

Using (2.1), one finds, after some straightforward algebra, 


$$
\begin{aligned}
F_{\beta} & =\frac{-2 H \beta R_{0} f_{0}}{R} \int_{0}^{2 \pi} \int_{0}^{R}\left(R^{3} / 6-R \frac{r^{2}}{2}+\frac{r^{3}}{3}\right) r d r d \theta \\
& =-\beta \pi H R_{0} f_{0} R^{4} / 10 .
\end{aligned}
$$

It is positive (southward) in the northern hemisphere for an anticyclone $\left(R_{0}>0 ; f_{0}>0\right)$ and negative for a cyclone.

\section{d) Detailed solution}

Combining (3.7) and (3.8), one ultimately finds our desired (surprisingly simple) migration formula,

$$
C=\left(\frac{3 \pi}{5} \beta V_{\max }\right)^{1 / 2} r_{\max }
$$

where $C$ is the minimum poleward speed for a cyclone (and the minimum equatorward speed for an anticyclone), $V_{\max }$ is the absolute value of the maximum velocity and $r_{\max }$ is the radius at which the velocity is a maximum $(R / 2$ in our analysis).

In the next section, we shall compare our analytical solution to our own numerical runs, to other numerical runs as well as to the lab experiments of FE. We shall see that, while (3.9) compares well to the numerical values, numerical mixing blurs the separating streamline to the extent that the detachment points cannot be easily identified. (This is also noted in the laboratory experiments of FE.) As expected, both the numerical and the laboratory speeds are lower than the 
analytically predicted speeds (3.9) because numerical and laboratory friction slows down the migration. This is the case even though (3.9) is the minimum predicted speed, because (3.9) is the minimum for an inviscid migration (i.e., when $v \rightarrow 0$ ), not a frictional migration.

\section{4. $\quad$ Numerical simulations}

We have used a single layer numerical model with a free surface (i.e., without the rigid lid approximation) that we adapted from the Black and Boudra original isopycnic model. The model has lateral Laplacian friction but no bottom and no vertical friction. We initialized it with a (10 km radius) stationary eddy with the previously discussed parabolic velocity profile (2.1). The numerical grid count was $101 \mathrm{X} 101, \Delta \mathrm{x}=\Delta \mathrm{y}=1 \mathrm{~km}$, and $\Delta \mathrm{T}=0.54 \mathrm{sec}$.

We conducted a total of 40 experiments with varying viscosity and Rossby numbers in an attempt to avoid eddy breakup and yet keep the viscosity at a minimum. We ultimately focused on four experiments described in Table 1; one of them is shown in Fig. 6. (Since our analytically predicted speed was constantly adjusted during the experiment, showing the four experiments requires four separate figures which we feel is un-necessary. The three that are not shown are almost indistinguishable from Fig. 6.) For each experiment, we measured the eddy center radius and speed everyday, implying that each experiment provides about 50 valid data points. The center of the eddy was identified as the point where the vorticity has its maximal value, whereas the radius is identified as the mean radius of the closed contour along which $\partial V_{\theta} / \partial r$ is a maximum. Although not really necessary, to make the plots easier to view, the center position was filtered with an 11 day centered running mean whereas the drift speed was filtered with a 21 day mean. The changes that the eddy experience with time due to the numerical friction are 
shown in Fig. 7. The streamlines in the vortex lee (adjusted for the eddy translation) are shown in Fig. 8, the balance of forces in Fig. 9 and the eddy trajectory is shown in Fig.10.

The run presented in Fig. 6 shows a good agreement between the predicted speed and the numerical speed after the eddy adjusts to the presence of $\beta$ (day 70 or so). The figure also displays good agreement with the numerical results consolidated by Smith (1993) to his empirical formula (dashed-line) and to the laboratory experiments of FE for intense eddies (that did not break up). Squares correspond to their squares (shown in their Fig. 9a) indicating a strong eddy whereas circles correspond to their circles indicating a still intense but somewhat weaker eddy. As expected, for intense eddies, the $\beta$-force is large so the meridional drift speed is large as well. In the same time, the lift is still relatively small because it is merely a function of the eddy orbital speed near the rim that is not strongly dependent of the eddy intensity and is supposedly very small in all cases. Hence, as shown in Fig. 9, intense eddies are expected to move toward the north whereas weaker eddies are expected to move more toward the northwest (due to an increased relative importance of the lift).

As should be the case, both the laboratory speeds and the numerical speeds are lower than our analytically predicted speed because both contain frictional forces that are absent from our model. Despite the approximations made in the derivation of the analytical formula, and despite the presence of friction in the numerics, the two values are consistently within $30-50 \%$ of each other for a long period of time (50 days or so) during which numerous calculations were made. Because of the unavoidable use of numerical friction that reduced the eddy intensity which increases the relative importance of the lift, the eddy meridional drift was merely three times as 
fast as the westward drift. This forced the eddy to migrate in the NNW direction rather than in the purely meridional direction (see Fig. 10, left panel). Although this is not an ideal situation, it is an acceptable comparison. Fig. 10 (right panel) clearly illustrates that the same NNW drift existed in the intense-eddies laboratory experiments of FE. While it is definitely possible to identify a region of very weak velocities at the lee of both the laboratory and numerical eddy (Fig. 8), because of a small but steady mixing of the eddy fluid with the environmental fluid, it is difficult to verify the detachment in the lee.

\section{Summary and Discussion}

We derived a simple analytical formula for very fast meridional migration rates of intense eddies with a strong barotropic component. The main balance of forces is conceptually analogous to that associated with the so-called "terminal velocity" of falling objects where the gravitational force balances the form-drag with the lift playing secondary role (Fig. 9). Here, the $\beta$-force balances the form-drag. We argue that most eddies probably do not have a barotropic component as it is quickly (10-100 days) dissipated by bottom friction. It is expected that eddies that do possess a barotropic component probably do so for only a relatively short time after their formation. Assuming that this bottom-driven dissipation is scaled as,

$$
H \cdot \frac{d u}{d t} \sim C_{D} u^{2}
$$


and that $C_{D} \sim 3 \times 10^{-3}, U \sim 0.2 \mathrm{~ms}^{-1}$ and $H \sim 4000 \mathrm{~m}$, we find that the dissipation time is roughly 75 days.

In this scenario, the eddies with the barotropic component will move primarily meridionally during the first 75 days of their life-time. For Agulhas rings, this certainly agrees with the observations of high northward speeds (Fig. 1), which can not be easily explained by neither Sverdrup advection nor bottom topography. Our migration speed formula, $\left(\frac{3 \pi}{5} \beta V_{\max }\right)^{1 / 2} r_{\max }$, suggests that these rings (with an $r_{\max }$ of, say, $40 \mathrm{~km}$, see e.g., Boebel et al. 2003, Ahran et al. 1999) will propagate toward the equator at a rate of $11 \mathrm{~ms}^{-1}$ or $9 \mathrm{~km} / \mathrm{day}$. This is also in agreement with the (cyclonic) observation that Fig. 1a displays. The cyclone in the North Atlantic (Fig. 1b) had a similar orbital speed but was considerably smaller. Taking $r_{\max }$ to be $30 \mathrm{~km}$ instead of $40 \mathrm{~km}$, we obtain a northward migration rate of about $7 \mathrm{~km} /$ day.

Overall, when our numerical experiments are compared to numerical experiments performed by others and to the laboratory experiments of FE, the results are satisfactory. Also, relative to the numerical speeds as well as the laboratory experiments (Fig. 6), the analytics always takes higher values due to the absence of friction. In addition, the streamlines in the eddy' lee show clear signs of a weakened flow downstream, consistent with the wake idea (Fig.8). The assumption of relatively small lift (for intense eddies) and, hence, small zonal movements is also verified by both our numerical runs and the laboratory experiments of FE (Fig. 10). However, identification of detaching streamlines was impossible due to unavoidable numerical mixing which blurs the eddy water with the outer water. 
As common for most numerical experiments of this nature (e.g., Chassignet and Cushman-Roisin 1991, Cherubin et al. 2006, Simmons and Nof 2002), we used viscosities corresponding to a diffusion speed $(v / \Delta x)$ of less than $1 \mathrm{~cm} / \mathrm{s}$. This and the values given in table 1 are roughly the same as those used by other investigators except in some cases (e.g., Hogan and Hulbrut 2000, Jacobs et al. 1999) where a bit lower values $\left(2 \mathrm{~m}^{2} / \mathrm{s}\right.$ instead of $\left.3 \mathrm{~m}^{2} / \mathrm{s}\right)$ could be used. This is because, in contrast to our model that has only horizontal friction, these models also include bottom and vertical friction as well as horizontal friction. Our frictional values are, of course, larger than what we wish we could use. They cause frictional effects to be noticeable within 50 days or so (Fig. 7), roughly the same time that it takes the eddy to adjust to $\beta$. These frictional effects reduced the eddy orbital speed, but their importance to the overall balance of forces in the north-south direction is negligible for all the experimental times. The reduction in the orbital speed can be easily seen from the upper panel of Fig. 7 whereas the insignificant role of friction in the force balance can be easily verified by examining the Reynolds number based on the migration speed $C$.

The Reynolds number measures the ratio of the inertial terms (form-drag in our case) to the horizontal frictional terms which, like brakes, push inward toward the eddy's rim attempting to slow both the orbital speed and the migration speed down. In the ocean, it is at least twoorders of magnitude larger than unity indicating that the direct effect of friction on the migration force balance is truly negligible. In the numerics, however, the Reynolds number is about three, indicating that the numerical frictional effects are not totally negligible.

The idealized force balance described here is offered as a possible explanation for the 
rapid transient meridional translation of some eddies; its actual role relative to other factors such as background flow mutual advection by other eddies and bottom topography remains to be explored in detail via further analysis of observations and very high-resolution general circulation models.

\section{6. $\quad$ Acknowledgements}

Eric Firing, the editor who processed this paper as well as two anonymous referees

provided very helpful and constructive comments. We acknowledge the support of the National Science Foundation Grants, OCE-0545204, OCE-0241036 and OPP/ARC-0453846 as well as NASA, NAG5-10860. 


\section{APPENDIX:}

$\beta \quad$ lineral variation on the Corolis parameter with latitude

$V_{m} \quad$ maximum orbital speed

$r_{m} \quad$ radius where the maximum speed occurs

$\psi \quad$ stream function

$\hat{h} \quad$ ring central depth

$R_{0} \quad$ Rossby number

$R \quad$ eddy radius

$C_{D} \quad$ non-dimensional bottom drag-coefficient

$h \quad$ total thickness

$u$ and $v$ horizonal velocity components

$H \quad$ the undisturbed depth

$\eta \quad$ free surface displacement

$B \quad$ Bernuolli integral

$g \eta \quad$ pressure term

$F_{D} \quad$ form-drag force

$u_{r} \quad$ radial velocity

$u_{\theta} \quad$ tangential velocity

$q \quad$ speed along the edge of the vortex

C migration speed (poleward for cyclone, equatorward for anticyclone)

f Corolis parameter

$v \quad$ viscosity 


\section{REFERENCES:}

Acheson, D. J. 1990. Elementary Fluid Dynamics. Clarendon Press, Oxford. pp. 397.

Arhan, M., Mercier, H., and Lutjeharms, J.R.E., 1999. The disparate evolution of three Agulhas rings in the South Atlantic Ocean. J. Geophys. Res., 104 (C9), 20,987-21,005.

Boebel, O., T. Rossby, J Lutjeharms, W. Zenk, and C. Barron, 2003: Path and variability of the Agulhas Return Current. Deep-Sea Res II, 50, 35-56.

Byrne, D. A. , Gordon, A. L., and Haxby, W. F., 1995. Agulhus Eddies: A synoptic view using Geosat ERM data. J. Phys. Oceanogr, 25, 902-917.

Chassignet, E. P. and Cushman-Roisin, B. 1991. On the Influence of a Lower Layer on the Propagation of Nonlinear Oceanic Eddies. Phys. Oceanogr., 21, 939-957.

Cherubin, L. M., Morel, Y. and Chassignet, E. P. 2006. Loop Current ring shedding: The formation of cyclones and the effect of topography. J. Phys. Oceanogr. 36, 569-591.

DeMaria, M., 1985. Tropical Cyclone Motion in a Nondivergent Barotropic Model. Mon. Wea. Rev, 113, 1199-1210.

Dewar, W. K. and Gailliard, C., 1994. The dynamics of barotropically dominated rings. J. Phys. Oceanogr, 24, 5-29.

Dewar, W.K. and M.Y. Morris, 2000. On the propagation of baroclinic waves in the general circulation. J. Phys. Oceanogr, 30, 2637-2649.

Firings, E., and Beardsley, R. C., 1978. The behavior of a barotropic eddy on a beta-plane. $J$. Phys. Oceanogr, 6, 57-65.

Flierl, G.R. 1984. Rossby wave radiation from a strongly nonlinear warm eddy. J. Phys. Oceanogr, 14, 47-58.

Flierl, G.R. Isolated Eddy Models in Geophysics. 1987. Annu. Rev. Fluid Mech, 19, 493-530.

Flór, J. B., and Eames, I., 2002. Dynamics of monopolar vortices on a topographic beta-plane. $J$. Fluid Mech., 456, 353-376.

Hogan, P. J. and Hulbrut, H. E. 2002. Impact of Upper Ocean-Topographical Coupling and Isopycnal Outcropping in Japan/East Sea Models with $1 / 8^{\circ}$ to $1 / 64^{\circ}$ Resolution. J. Phys. Oceanogr, 30, 2535-2561.

Jacobs, G.A., Hogan, P. J. and Whitmer, K. R. 1999. Effects of eddy variability on the circulation of the Japan/East sea. J. Oceanogr, 55, 247-256. 
Kamenkovich, V. M., , Koschlyakov, V. N. and Monin, A. S., 1986. Synoptic eddies in the ocean, Reidel, Dordrecht, Holland. pp. 433.

Killworth, P. D. and Blundell, J. R. 2004. The dispersion relation for planetary waves in the presence of mean flow and topography: II. Two-dimensional examples and global results. $J$. Phys. Oceanogr, 35, 2110-2133.

Korotaev, G. K., 1997. Radiating Vortices in Geophysical Fluid Dynamics. Surv. Geophys., 18, 567-619.

McWilliams, J. C. and Flierl, G. R. 1979. On the evolution of isolated, nonlinear vortices. $J$. Phys. Oceanogr. 9, 1155-1182.

Mied, R. P. and Liendeman, G. J. 1979. The Propagation and Evolution of Cyclonic Gulf Stream Rings. J. Phys. Oceanogr, 9, 1183-1206.

Nof, D. 1983. On the migration of isolated eddies with application to Gulf Stream rings. J. Mar. Res, 41, 399-425.

Nof, D. 1985. Joint vortices, eastward propagating eddies and migratory Taylor columns. $J$.

Phys. Oceanogr, 15, 1114-1137.

Nof, R. and Simon, L., 1987. Laboratory experiments on the merging of anticyclonic eddies. $J$. Phys. Oceanogr, 17, 343-357.

Paldor, N., S. Rubin and A.J. Mariano. 2006. A consistent theory for linear waves of the Shallow Water Equations on a rotating plane in mid-latitudes. J. Phys. Oceanogr, In Press.

Pichevin, T, Nof, D, and Lutjeharms, J. 1999. Why are there Agulhas Rings. J. Phys. Oceanogr. 29, 693-707. |

Reznik, G.M. and W.K. Dewar, An analytical theory of distributed axisymmetric barotropic vortices on the $\beta$-plane. J. Fluid Mech, 269, 1994, 301-321.

Reznik, G.M., and Grimshaw, R. 2001. Ageostrophic dynamics of an intense localized vortex on a beta-plane. J. Fluid Mech, 443, 351-376.

Reznik, G.M., Grimshaw, R and Benilov, E. S. 2000. On the long-term evolution of an intense localized divergent vortex on the beta-plane. J. Fluid Mech, 422, 249-280.

Robinson, A.R. 1983. Eddies in Marine Science. Springer-Verlag, Berlin Heidelbeg, pp. 609.

Rossby, C. G. 1948. On displacements and intensity changes of atmospheric vortices. J. Mar. Res, 7, 175-187. 
Schmid, C, Boebel, O, Zenk, W, Lutjeharms, JRE, Garzoli, SL, Richardson, PL and Barron C. 2003. Early evolution of an Agulhas Ring. Deep-Sea Res, 50 (1), 141-166.

Simmons, H. and Nof, D. 2002. The squeezing of eddies through gaps. J. Phys. Oceanogr, 32, 314-335.

Smith, R. B. 1993. A Hurricane Beta-Drift Law. J. Atmos. Sci, 50, 3213-3215. 


\section{Captions List:}

Table 1: Details of the primary four experiments. Note that the viscosity gives a decay time scale $\left(r_{\max }^{2} / v\right)$ of $50-80$ days, roughly the same time as the eddy adjustment to $\beta$ ( approximately 70 days) implying that the analytically predicted speed needs to be adjusted throughout the experiment.

Fig. 1a: Path of typical Agulhas rings. Steric anomaly at $500 \mathrm{dbar}$ is contoured. The tracks are smoothed from estimates of the eddy center, with shapes at evenly spaced time intervals of 34 days. Adapted from Byrne et al. (1995). Note that, initially, the rings drift fast to the NNW. After about 100 days, thier migration rate slows down considerably and their migration path becomes more and more zonal.

Fig. 1b: Successive positions of a single cyclonic eddy in the POLYMODE region on (I) December 27-30, 1977, (II) January 17-19, 1978 for the plot and (III) February 9-10, 1978 (adapted from Kamenkovich et al. 1986 who credit Ivanov and Paramonov, 1980 for the plot). The contours represent the depth isolines (in meters) of the $15^{\circ} \mathrm{C}$ isothermal surface. (Larger orbital speeds and larger migration rates at a different orientation were observed after the shown periods. This suggests that, in the end of February, the eddy probably interacted with another feature.)

Fig. 2: A conceptual picture of our simplified Agulhas rings formation process. The left panel shows a one-layer ocean [with density $(\rho+\Delta \rho)$ into which a finite amount of lighter fluid (with 
negative relative vorticity) is injected (on the surface). Once the injection is completed, (right panel), there are anticyclones in both layers as the lower layer is squeezed. Note that, strictly speaking, conservation of potential vorticity and angular momentum imply that there will also be anticyclonic motion outside the lens boundary because the lower fluid is forced outward. For simplicity, we shall neglect these outside motions and consider parabolic velocity profiles in both layers, i.e., neither the lens nor the barotropic component below correspond to uniform potential vorticity. Furthermore, our parabolic velocity profile (Fig. 4) does not exactly correspond to a solution of the injection problem posed above. The derivation of such a solution is straightforward but cumbersome (see e.g., Nof and Simon, 1987) and is really not necessary for our migration rate calculation.

Fig. 3: The same as Fig. 2 except for the North Atlantic cyclone. Here, a finite amount of light fluid is sucked out of the upper layer forming a cyclone behind it. A cyclone is also formed underneath because the lower layer thickness is stretched. Again, for simplicity, we shall take both layers to have a parabolic orbital velocity profile (Fig. 4) even though such a profile does not correspond to uniform potential vorticity and does not exactly correspond to an exact solution of the conceptual suction process.

Fig. 4: The adopted simplified structure of the vortices shown in Figs. 2, and 3. We take the two eddies situated on top of each other as one barotropic vortex extending all the way from the surface to the bottom. The orbital velocity profile is parabolic with the velocity reaching a maximum at $R / 2$ and vanishing along the edge $(R)$. 
Fig. 5: Schematic diagram of the northward propagating (southern hemisphere) anticyclone (Agulhas) or the (northern hemisphere) cyclone in a coordinated system traveling with the vortex at the speed $C$. The barotropic eddy scale $(\sim 100 \mathrm{~km})$ is much smaller than the barotropic Rossby radius $(\sim 2000 \mathrm{~km})$ so the upstream flow surrounding the vortex is viewed as inviscid. To estimate the maximum drag on the vortex, it is assumed that this flow separates from the vortex western and eastern most edges (A and B) and that the flow is very weak in the lee (shaded region). The surrounding flow in the upstream section (i.e., for $y \geq 0$ ) is taken to be potential flow with no vorticity and no-circulation. The sea-level differences noted were obtained by applying the Bernoulli along the streamline originating upstream at infinity $(x=0, y \rightarrow \infty)$ and passing through the stagnation point $\mathrm{O}$, and the detachment points $\mathrm{A}$ and $\mathrm{B}$.

Fig. 6: The adjusted analytical drift (upper solid curve) and numerical (lower curve) meridional speed as a function of time for experiment \# 1. The adjustment to the analytical speed was made on the basis of both the numerically measured maximum speed and the radius. The dashed line is the empirically predicted speed of Smith (1994). The squares and circles are the results of the laboratory experiments of FE (their figure 9a). Note that the experimental adjustment time $\left[(\beta R)^{-}\right.$ ${ }^{1}$ ] is roughly 70 days so that the period that we need to focus on is the period between 70 and 115 days. For that period, the theoretical and numerical values compare to each other fairly well despite the presence of friction in the numerics and the resulting (unavoidable) presence of some zonal speed (Fig. 8). As expected, the theoretical meridional speeds are higher than the numerical speeds which are slowed down by friction. Note that the numerical measurements were done daily so each experiment corresponds to 50 or so data points. 
Fig. 7: The numerical decrease in Rossby number (upper panel) and the increase in eddy size (lower panel) as a function of time. Both reflect the effect of horizontal friction, which, due to numerical stability issues common for all numerical experiments of this nature (e.g., Cherubin et al. 2006, Simmons and Nof 2002), could not be placed at less than 5-9 $\mathrm{m}^{2} / \mathrm{s}$ (corresponding to an acceptable diffusion speed of $1 \mathrm{~cm} / \mathrm{sec}$ or less).

Fig. 8: Streamlines (viewed from the moving eddy) for our first numerical experiments (left) and a corresponding laboratory experiment of FE (right). Although the detachment points cannot be seen due to either numerical (our case) or actual mixing (FE case), a region of weak flow is identified in the lee. Also, the noted asymmetry in the direction perpendicular to the migration indicates some friction-induced lift (neglected in our analytical calculation) which forces the eddy to migrate in the NNW direction, instead of directly due north. (Adapted from FE, figure 6d.)

Fig. 9: Schematic diagram of the balance of forces with (right) and without lift (left). For the lift to exist some orbital motions from the eddy' core must somehow be transmitted (via friction) to the outer fluid. Since the eddy motions along the rim are small, this transmittal is expected to be small and negligible and this is what our analytics address (left panel). In reality, however, there will be some orbital motion transmission and, in the case of a cyclone in the northern hemisphere, the orbital motions will speed up the outer fluid motions in the west and slow them down in the east giving rise to a westward lift. This lift forces the eddy migration to tilt in the 
NNW direction so that the $\beta$-force will have a component balancing the lift (right panel). It is expected that, relative to the northward $\beta$-force, the westward lift will be small for intense eddies and large in the weak eddies case.

Fig. 10: Left panel: The trajectory of the eddy center (maximum relative vorticity) for experiment 1 and 2. For cosmetic reasons, the center position is calculated daily and then filtered with an 11 day centered running mean. Solid black dots are plotted every 10 days starting at $\mathrm{t}=$ 0. Right panel: The analogous laboratory experiments of FE for two intense eddies; see their figure 9a (from which this figure was adopted) for the parameters associated with the circles and squares. Note the similarity in the trajectories. 